\title{
Follow-up Evaluation of Psychotherapy Protocols for Women with a History of Intimate Partner Violence: Scoping Review
}

\author{
Laura Nichele Foschiera $^{1}$ (D) - Melina Friedrich Dupont ${ }^{1}$ (D) . \\ Luísa Fernanda Habigzang ${ }^{1}$ (D)
}

Accepted: 29 October 2021 / Published online: 24 November 2021

(c) Associação Brasileira de Psicologia 2021

\begin{abstract}
To investigate the methodological aspects, main results, and limitations of the follow-up evaluations of psychotherapeutic interventions for women with a history of intimate partner violence (IPV). A search was carried out in the SciELO, Scopus, PsycINFO, and Web of Science databases, using the descriptors: ("clinical trial" OR "therapy" OR "psychotherapy" OR "psychological treatment") AND ("violence" OR "mistreatment" OR "intimate partner violence" OR "domestic violence" OR "conjugal violence") AND ("women"). 1480 articles were retrieved and after applying the inclusion and exclusion criteria, 11 articles composed the final sample. The majority of the follow-up assessments were carried out through the reapplication of the basic instruments, with the period of 3 months post-intervention being the most used. Sample losses were identified in all the studies analyzed. The limitations and difficulties were associated with the complexity of the target population. This review identified the importance of the follow-up evaluations and the need to adapt them to ensure the effectiveness of the interventions, aiming to reach a larger number of women, strengthen protective aspects, and prevent revictimization.
\end{abstract}

Keywords Intimate partner violence - Women · Psychological interventions · Clinical trial protocols $\cdot$ Follow-up studies

\section{Introduction}

According to the World Health Organization (WHO, 2019), violence against women is defined as any act of gender-based violence that results in physical, sexual or mental suffering for women, and can be carried out by anyone. Violence against women

Luísa Fernanda Habigzang

habigzang.luisa@gmail.com

1 Pontifícia Universidade Católica Do Rio Grande Do Sul, Porto Alegre/Rio Grande Do Sul, Brazil 
is considered a violation of human rights, and a public health problem. Currently, it is estimated that one in three women worldwide has suffered physical and/or sexual violence, and among the forms of violence against women, intimate partner violence is the most common (WHO, 2020). Intimate partner violence (IPV) is a specific form of violence against women, in which acts of physical, sexual or psychological violence are committed by current or former intimate partners (WHO, 2019). Data from the World Health Organization indicate that $30 \%$ of women in the world that have had an affective relationship report this violence and 38\% of homicides of women are committed by current or former intimate partners (WHO, 2019; 2020).

The experience of IPV is a risk factor for women's mental health, who, after suffering violence, tend to be more likely to develop depression, emotional regulation problems, anxiety, post-traumatic stress disorder, suicidal ideation, and substance use disorders, as well as problems in physical, sexual, and reproductive health (Bacchus et al., 2018; WHO, 2019; Zancan \& Habigzang, 2018). Due to the high rates of IPV, studies have sought to develop alternative interventions to mitigate the main psychological consequences that women can develop after the abusive experience (Habigzang et al., 2018; Matud et al., 2014). Many studies have presented positive results, such as decreased levels of depression and anxiety (Habigzang et al., 2018; Matud et al., 2014) and post-traumatic stress disorder (PTSD) symptoms (Matud et al., 2014), which highlight psychotherapy as an important resource for the health promotion of women with a history of IPV. A psychological intervention is defined as an action aimed at interfering with something, which seeks to stop or modify a certain process (American Psychological Association [APA], 2017). Psychological interventions comprise psychotherapy, a process in which the therapist seeks to help the patient to find emotional relief, the solution to a specific problem or the modification of their way of thinking, feeling and behaving (APA, 2021). Considering the complexity of the violence phenomenon, it is recommended that psychotherapy is anchored in scientific evidence of efficacy and effectiveness, promoting psychological treatments with the application of empirically proven techniques and practices in terms of assessment and intervention (Berg, 2019).

Evidence-based practice is considered an approach that seeks to promote patient health through treatment based on the best available scientific evidence, in accordance with clinical expertise and the characteristics of the patient, their requirements, and context (Rousseau \& Gunia, 2016). Randomized clinical trials, systematic review studies, and meta-analyses supply evidence of the effectiveness of interventions, providing scientific support for professionals seeking specialized knowledge about a certain phenomenon (Berg, 2019; Melnik et al., 2014).

Through the analysis of randomized clinical trials of psychotherapy, a systematic review investigated several psychotherapeutic approaches for women with a history of IPV, finding evidence for the effectiveness of Cognitive-Behavioral Therapy (CBT) for this population (Hameed et al., 2020). Similarly, a meta-analysis analyzed brief psychological interventions for women with a history of IPV, indicating CBT as the most effective approach (Arroyo et al., 2017). Recently, a systematic review specifically investigated CBT protocols for women with a history of IPV, reinforcing the evidence for the effectiveness of this approach in the treatment of this population (Petersen et al., 2019). 
To confirm the effectiveness of interventions with women with a history of IPV, it is necessary to carry out follow-up assessments, which means reassessing the symptoms after a certain period of time to verify whether the therapeutic effects are maintained in the medium and long term. Regarding the format of the follow-up assessments, it is possible to use the same scales as the pre-test assessments, in addition to structured or unstructured interviews. The most frequent format in the literature is the reapplication of the instruments that comprised the pre-test evaluation, with the application period varying between 1 week and 12 months, which can be performed more than once (Cort et al., 2014; Crespo \& Arinero, 2010; Matud et al., 2014).

However, most studies that develop interventions with women with a history of IPV face challenges in assessing effectiveness in the medium and long term. Among them, sample limitations due to treatment abandonment, as well as methodological difficulties related to the implementation of the intervention program can be highlighted (Hardesty \& Olgosky, 2020). These factors can impact the performance of follow-up assessments, making it difficult to produce evidence of the effectiveness of the proposed interventions with this population.

A gap in the literature about the particularities concerning follow-up evaluations was identified: most articles about psychological interventions for women with IPV history focus on the treatment phases and the intervention techniques, but lack a thorough description of the follow-up evaluation, failing to properly report about its methodology and particularities. Such remarks highlight the need to broaden this research topic, in order to obtain theoretical and technical knowledge about the most effective practices for follow-up evaluations. Follow-up studies are key for a proper evaluation of evidence-based psychotherapy protocols, which are particularly important in the treatment of women with IPV history. In this sense, this study aims to investigate the methodological aspects, main results and limitations of the followup evaluations of psychotherapeutic interventions for women with IPV history.

\section{Method}

\section{Data Collection Procedures}

The authors independently consulted four databases: SciELO, Scopus, PsycINFO, and Web of Science. The string used for the search was: ("clinical trial" OR "therapy" OR "psychotherapy" OR "psychological treatment") AND ("violence" OR "mistreatment" OR "intimate partner violence" OR "domestic violence" OR "conjugal violence") AND ("women"). The database search period was from June 1 to 8, 2020. A total of 1480 articles were found, of which 7 were in SciELO; 714 Scopus; 185 PsycINFO; and 574 in the Web of Science.

The results were imported into the Zotero (Corporation for Digital Scholarship) software. Through the program, 364 duplicates were identified among the databases. For the sample selection process, the results were imported into the Rayyan QCRI (Qatar Computing Research Institute) software, which is specific for carrying out review studies. After importing the data, the Rayyan software identified another 12 duplicates among the results, which left 1104 articles. 
While reading the abstracts, the judges included articles based on five criteria (IC): (1) scientific articles published in the area of mental health; (2) published in Portuguese, English or Spanish; (3) published between the years 2015 and 2020; (4) empirical reports evaluating psychotherapy protocols with follow-up evaluations for women with a history of IPV; and (5) those that included the descriptors used in the title, abstract or keywords. The following exclusion criteria (EC) were also applied: (1) book chapters, dissertations, theses, abstracts and works presented in congresses; and (2) case studies. After applying all the criteria, 1093 articles were excluded. The reasons for exclusion were: 2 were not published during the specified period; 667 were not psychotherapy protocols; 246 were not empirical; 60 were case studies; 51 were books or book chapters; 30 were not specific for women with a history of IPV; 24 were theses or dissertations; 5 did not have follow-up assessments; and 3 were works presented in congresses. In addition, 1 article was excluded because it did not have an abstract available, making it impossible to apply the inclusion and exclusion criteria. A total of 16 articles were considered eligible for full reading. After reading in full, five article were excluded: 2 because they were not performed by health professionals (e.g. psychologists or psychiatrists); 2 for not presenting follow-up assessments; and 1 due to presenting only the description of the intervention protocol, without the results. Therefore, the final sample consisted of 11 articles (Fig. 1).

\section{Analysis Procedures}

A descriptive synthesis of the methods used in the studies for the follow-up evaluations was carried out. The synthesis included: sample size; description of the

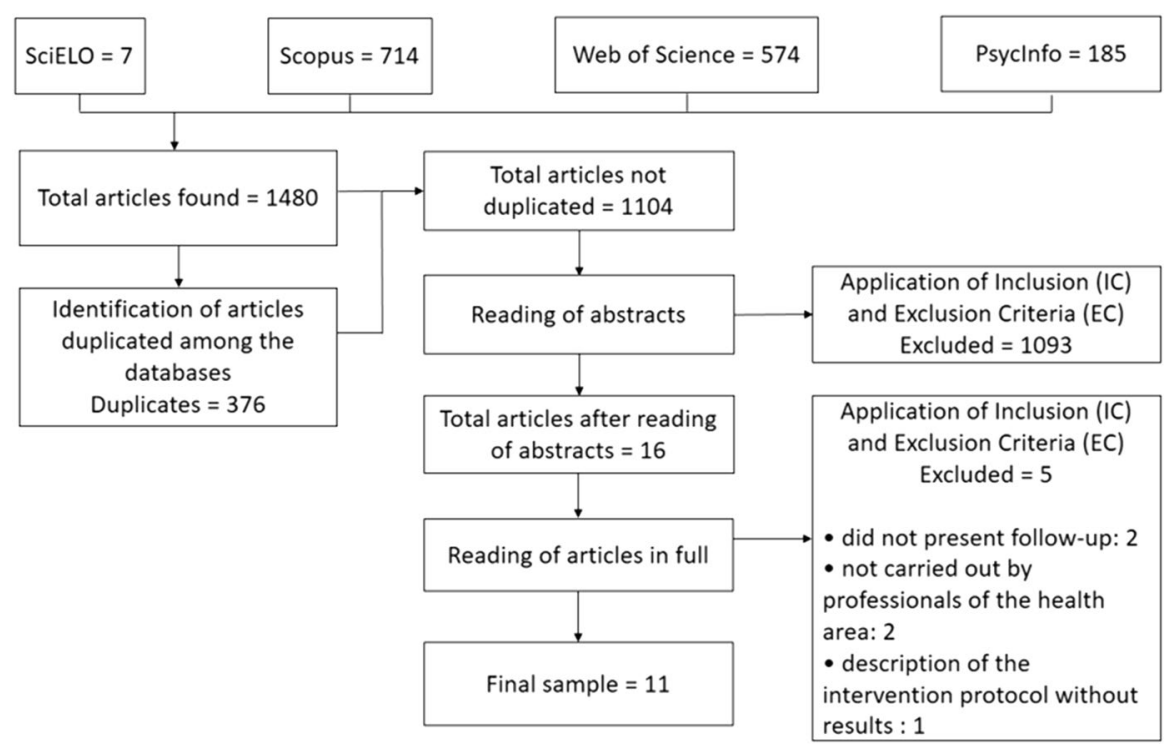

Fig. 1 Flow chart of the sample selection 
evaluation format and instruments used; frequency of follow-up assessments; and main therapeutic effects. The analysis and extraction of this information was carried out by two independent experts. In case of disagreement, a third expert evaluated the article. Content analysis was also carried out (Bardin, 2011), aiming to identify the main themes associated with the challenges and difficulties in conducting followup evaluations with women victims of IPV. The analysis was conducted through the reading and extraction of words associated with the characteristics of the content. Subsequently, semantic categorization and grouping of the words into content themes were performed.

\section{Results}

The 11 studies came from the following countries: United States $(n=4)$, Spain $(n=2)$, Portugal $(n=1)$, China $(n=1)$, Colombia $(n=1)$, India $(n=1)$ and Iran $(n=1)$. The age range, mean, and standard deviation of the participants in each study are displayed in Table 1 . The studies included in this review were evaluated in order to comprehend the structure of the follow-up assessments, as well as the therapeutic effects obtained with the intervention (Table 1).

The results of the analyses showed variation in the format of the follow-up assessments: four studies conducted semi-structured interviews to assess the symptoms of PTSD and history of IPV, combined with the reapplication of the same instruments used in the pretest measures of the intervention (Allard et al., 2018; Johnson et al., 2016; Matud et al., 2016; Orang et al., 2017); six studies carried out the follow-up assessment only with the reapplication of the base instruments (Beck et al., 2016; Choi et al., 2018; Naismith et al., 2020; Patel et al., 2019; Santos et al., 2017; Tirado-Muñoz et al., 2015); and one study conducted a fully qualitative interview at the follow-up (Trabold et al., 2017).

The analysis of the studies identified the main assessment instruments and clinical outcome measures used in the follow-up assessments. The majority of the studies evaluated symptoms of depression $(n=8)$ and history of past and current intimate partner violence $(n=8)$. Other symptoms evaluated were PTSD $(n=7)$, perception of quality of life $(n=4)$, self-esteem $(n=4)$, social support $(n=4)$, guilt $(n=3)$, anxiety $(n=3)$, and shame $(n=1)$. One study evaluated post-traumatic memories and cognitions (Beck et al., 2016) and another study investigated the maintenance of beliefs about conjugal violence during the follow-up period (Santos et al., 2017). Two studies evaluated the participants' satisfaction with the intervention performed (Johnson et al., 2016; Trabold et al., 2017), one study evaluated the therapeutic alliance (Naismith et al., 2020) and another verified the participants' progress throughout the psychotherapy (Santos et al., 2017). The study in which the follow-up presented an exclusively qualitative methodology explored aspects associated with current relationships, the occurrence of new situations of IPV, the participants' perceptions about the content worked on during the intervention and whether the women entered into a new therapeutic service after the end of the intervention (Trabold et al., 2017). 

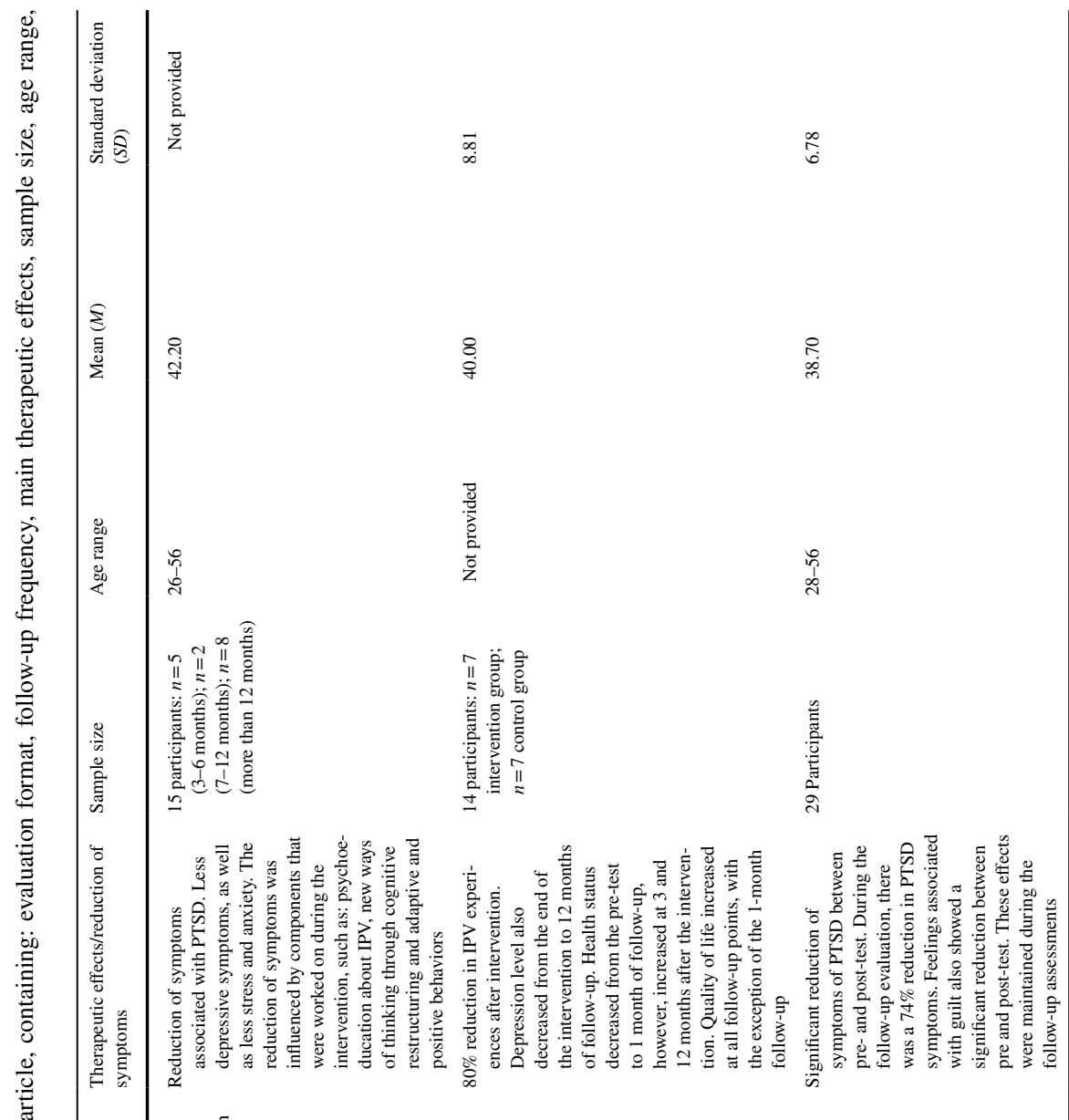

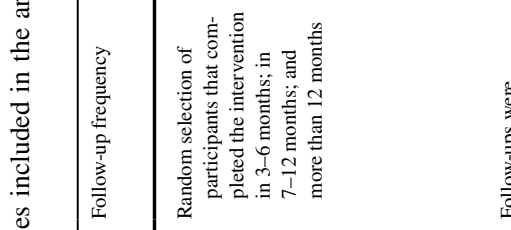

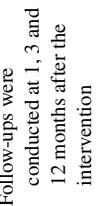

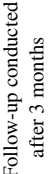
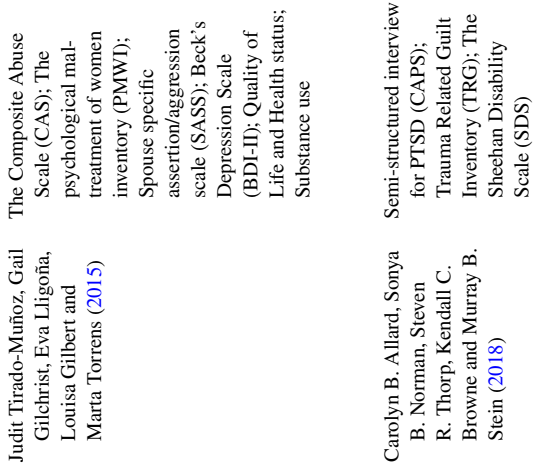


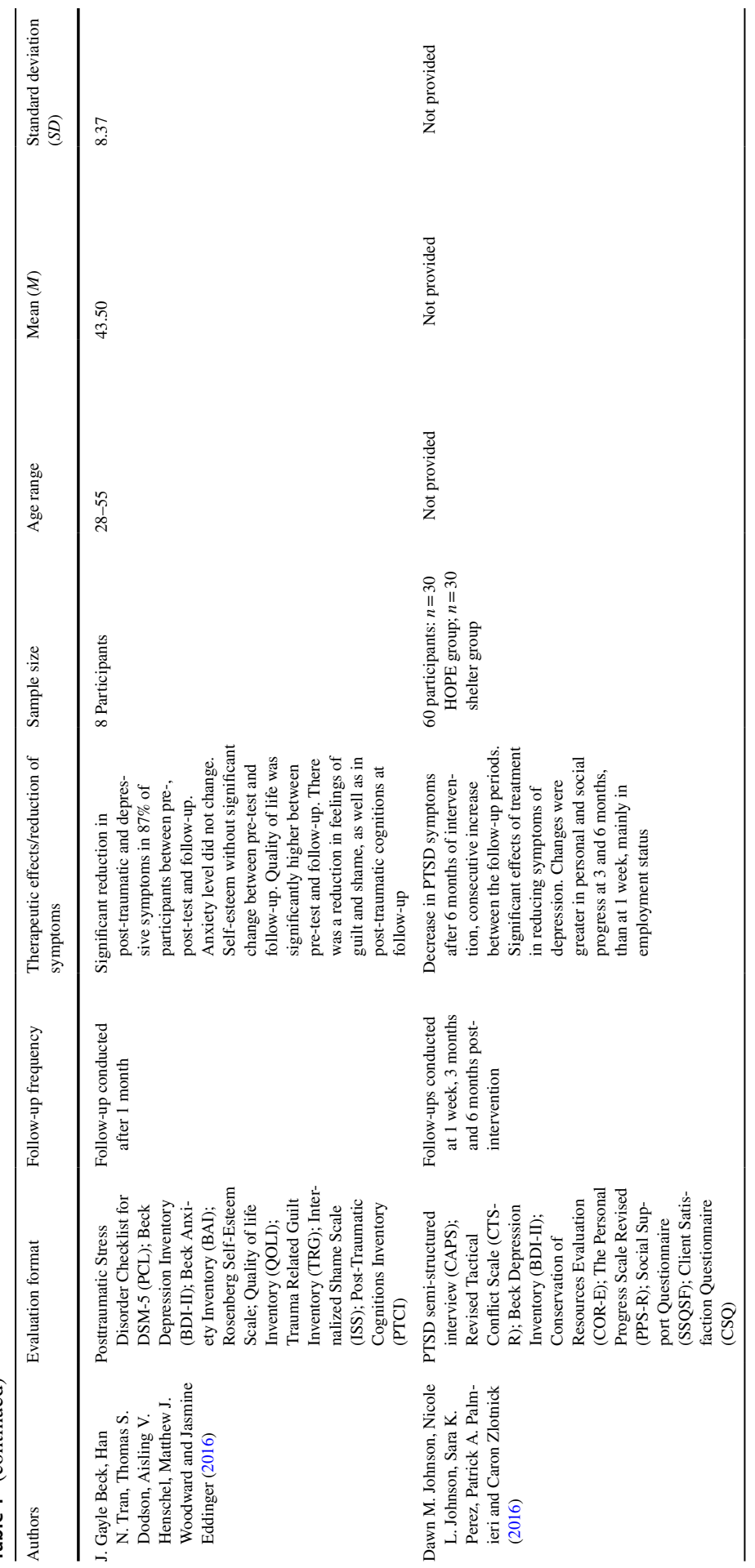




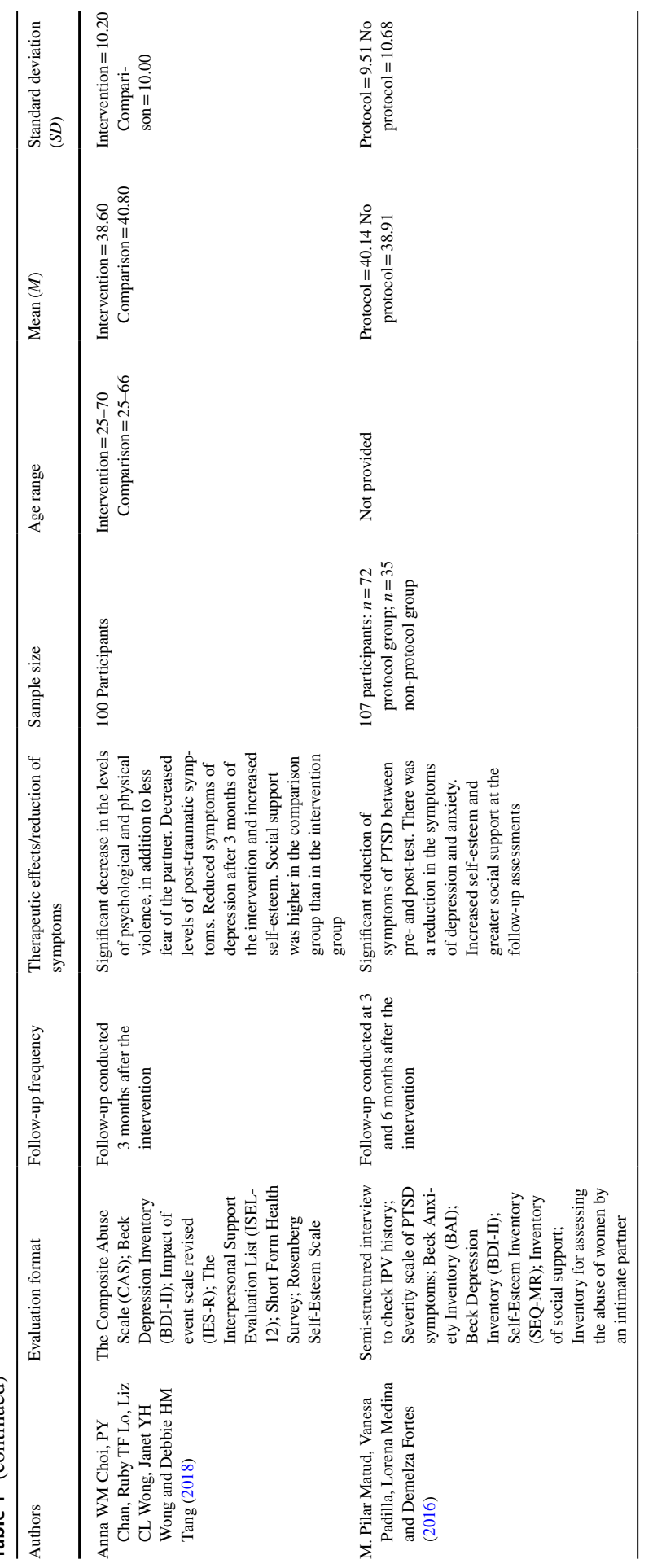




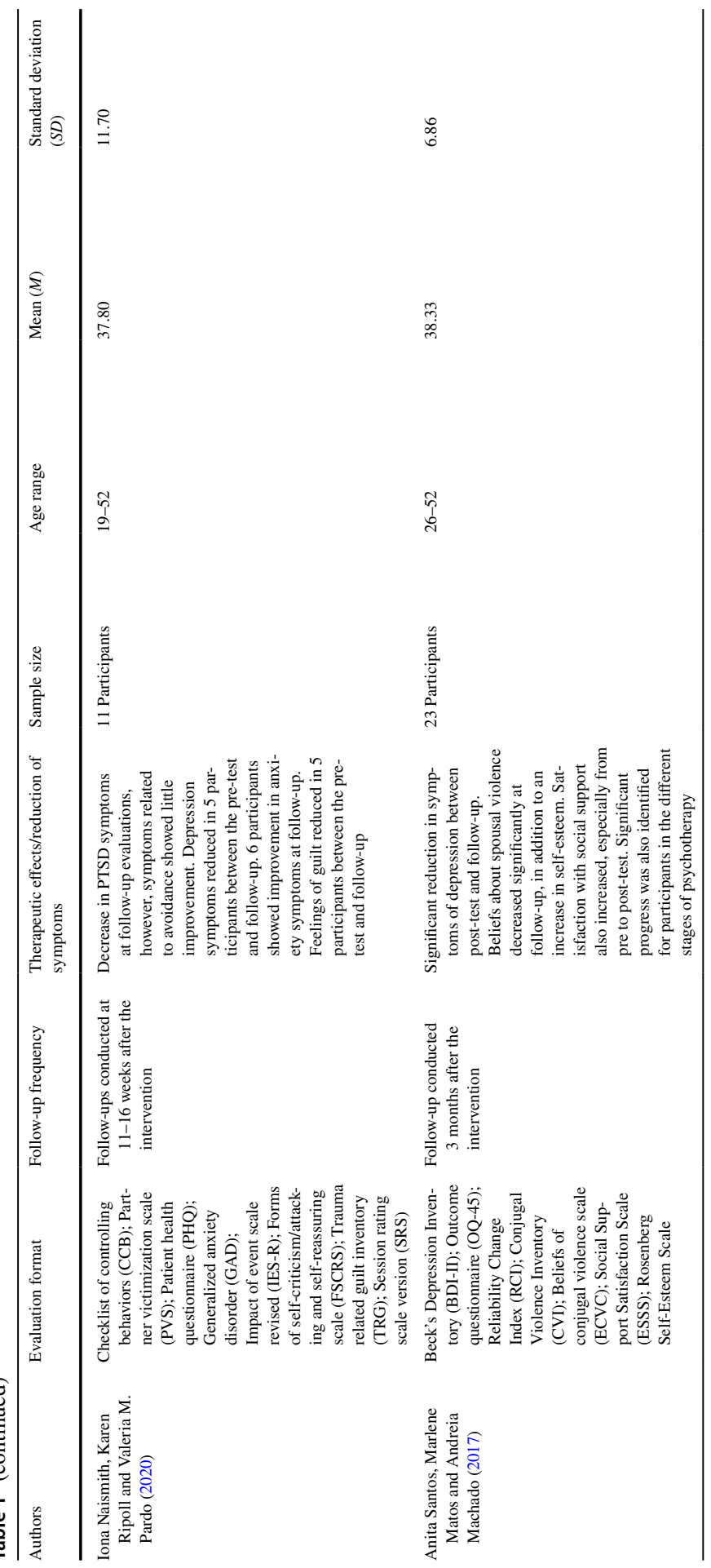




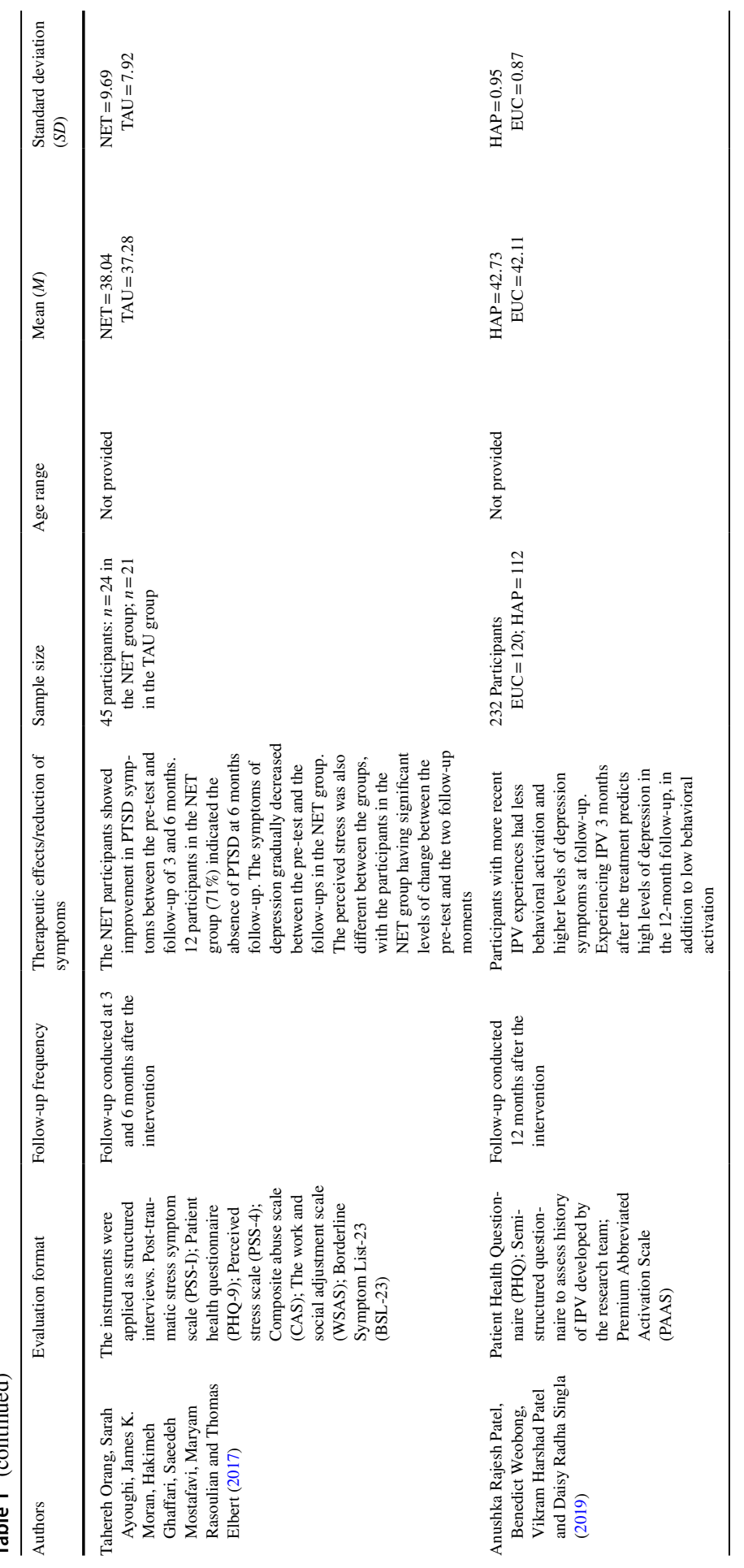


The interval between the end of the intervention and the performance of the followup assessments varied between 1 week (minimum) and more than 12 months (maximum). Most of the studies conducted a follow-up after three months $(n=9)$. The next two most frequent follow-up intervals were 6 months $(n=4)$ and 12 months $(n=3)$. Only two studies described the access to participants in the follow-up: both chose to preferably perform the assessment in person, although it could be done by telephone, if it was not possible for the participant to attend the place (Naismith et al., 2020; Tirado-Muñoz et al., 2015). Four studies reported offering the participants a financial incentive, usually after completing each stage of the study (Allard et al., 2018; Johnson et al., 2016; TiradoMuñoz et al., 2015; Trabold et al., 2017). Values ranged from 20 to US\$400. The sample flow also varied between studies. Two studies had expressive samples that completed the follow-up evaluations $(n=100)$ (Choi et al., 2018; Patel et al., 2019). The flowchart and sample loss in the follow-up of the studies are shown in Fig. 2.

One of the reasons associated with the gradual sample loss of participants in the studies involves the delimitation of the inclusion criteria. Five studies (Allard et al., 2018; Beck et al., 2016; Johnson et al., 2016; Santos et al., 2017; Orang et al., 2017) established the diagnosis of PTSD (partial or total) as an inclusion criterion. Regarding the relationship status, only two studies had requirements to include participants that were already separated from the aggressors and with no intention of resuming the relationship (Allard et al., 2018; Beck et al., 2016). Regarding the participant's current relationship status, one of the inclusion criteria in three studies was that the participant still be in the relationship (Orang et al., 2017; Patel et al., 2019; TiradoMuñoz et al., 2015); in four studies, the women had to be in the relationship or have had recently left an abusive relationship (Johnson et al., 2016; Matud et al., 2016; Naismith et al., 2020; Santos et al., 2017), and in two studies, there were no specific requirements regarding the participant's current relationship status (Choi et al., 2018; Trabold et al., 2017). Two studies conducted interventions with women in situations of extreme vulnerability, in addition to their own history of violence: Johnson et al. (2016) conducted the study with women in institutional care, and Choi et al. (2018) with refugee women.

The therapeutic effects or symptomatic reductions were outcomes explored by all the studies. The interventions that assessed PTSD symptoms identified a significant reduction in symptoms between the pre-test and follow-up. In addition, some studies indicated that the therapeutic effects were successive between the follow-up periods, with the longer intervals (e.g. 6 and 12 months) presenting more evident improvements (Johnson et al., 2016; Matud et al., 2016). In the study conducted by Orang et al. (2017), the researchers showed that 12 participants (71\%) from the Narrative Exposure Therapy (NET) group demonstrated the absence of PTSD symptoms in the 6-month follow-up evaluation, with significant effect size (Hedges' $g=1.77$ ). In the study carried out by Naismith et al. (2020), the follow-up interval was 11 to 16 weeks (between 3 and 4 months) and the results also showed reductions in posttraumatic symptoms. However, symptoms associated with avoidance showed little improvement.

Other studies also identified significant effects of the treatment between the preand post-test, and in the follow-up period. Allard et al. (2018) reported total remission of PTSD symptoms in $63 \%$ of participants after 3 months of treatment. They 
identified a considerable effect size between pre- and post-test in relation to the feeling of guilt (Cohen's $d=2.18$ ). Matud et al. (2016) also identified considerable clinical significance in their results: the study demonstrated that, in the intervention group, $47.8 \%$ of the participants had a diagnosis of PTSD before starting treatment, which decreased to $14.3 \%$ in the follow-up at 6 months. Symptoms of depression also decreased from $71.4 \%$ at the beginning of treatment, to $8.4 \%$ after 6 months. The clinical outcomes of all the studies included are described in Table 1.

Another objective of this review article was to present the difficulties and challenges faced by the researchers when conducting interventions with women victims of IPV, through a content analysis. Two main categories of content associated with the follow-up were identified: (1) sample losses; and (2) methodological limitations. Concerning the sample losses, the majority of the studies showed difficulties in the recruitment and follow-up stages, with a high percentage of dropouts (i.e. women abandoning the treatment) throughout the intervention. Some factors can be associated with losses during the follow-up period, such as the study inclusion criteria. The studies that requested that the women were with their intimate partner during the intervention presented losses at follow-up. In the study by Tirado-Muñoz et al. (2015), 14 women started the intervention ( $n=7$ intervention group; $n=7$ control group). In the intervention group, there was no loss during the follow-up assessments. However, in the control group, only two women performed the follow-up. The study of Patel et al. (2019) also showed losses at the 12-month follow-up assessments. In the enhanced usual care group, nine women did not perform the follow-up ( $n=120$ started; $n=111$ finished), and in the healthy activity program group, 12 women did not perform the evaluation ( $n=112$ started; $n=100$ finished).

The methodological limitations category mainly refers to the challenges of conducting psychotherapeutic interventions with a control group. In the studies analyzed, limitations associated with assessment measures were presented, mainly due to the use of self-report instruments rather than more controlled

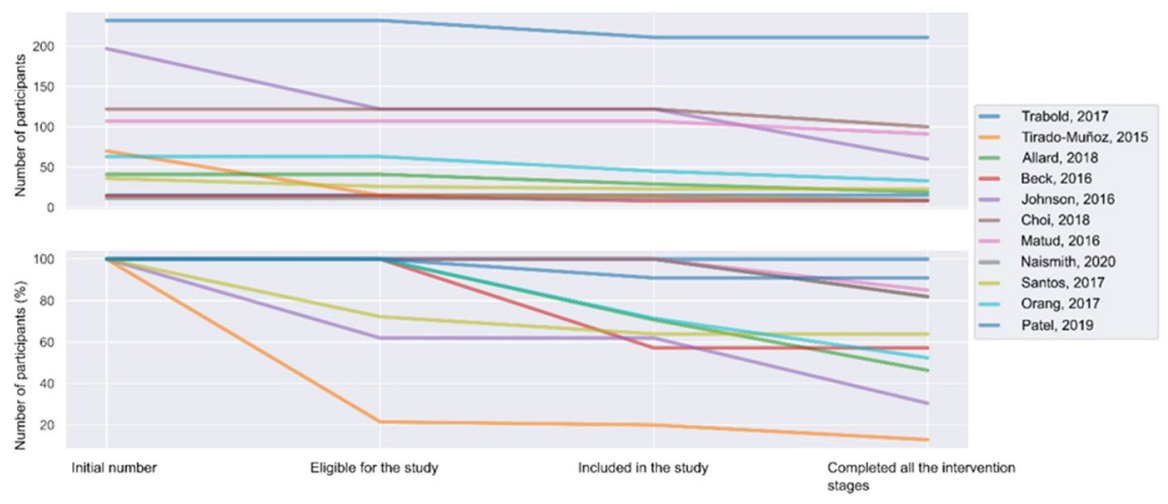

Fig. 2 Number of participants in each study phase. Note. Sample flowchart of the studies. The recruitment stages, sample selection and number of concluding participants are represented on the horizontal axis. The upper graph presents the absolute number of participants in each phase. The lower graph presents the number of participants converted to percentage 
observational methods. In addition, many studies highlighted the challenge of conducting longitudinal studies with women victims of IPV, as contact can be easily lost: most women face risky situations and legal issues, and they often need to carry out changes of residence, work and telephone number. In the study by Matud et al. (2016), the most reported reasons for interrupting the intervention were: starting a new job, incompatible schedules, change of residence, difficulties in the care of children during the intervention, and financial problems that prevented them travelling to the place. Another factor referring to methodological limitation is the access to participants after the completion of the intervention. Due to different restrictions, mainly financial, many participants faced difficulties in travelling to the location, preventing the performance of the follow-up assessment.

The follow-up interval was also highlighted in the articles as an important methodological limitation. Many of the studies reported the difficulty of implementing longer intervals with the participants, mainly due to the sample-related challenges described above. The study by Choi et al. (2018), for example, showed that, despite the reduction in symptoms between the pre-test and follow-up, just 3 months of follow-up was not sufficient to verify the long-term effectiveness of the treatment. Santos et al. (2017) also identified that the symptomatological reduction was not totally significant in the follow-up and indicated the need for more prolonged interventions for women with a history of IPV. The difficulty in verifying the effectiveness of the intervention between the follow-up intervals can also be associated with the participants' diagnosis of PTSD, especially if they remained in the abusive relationship. In the study by Orang et al. (2017), analyses were carried out to verify whether IPV experiences that occurred during the intervention and in the follow-up periods (e.g. 3 and 6 months) had an impact on the PTSD symptoms, both for the NET group and for the group with usual treatment. Intimate partner violence during the intervention or in the follow-up period had a significant impact on PTSD symptoms in both groups. Nevertheless, the participants in the NET group had reduced post-traumatic symptoms when compared to the group with usual treatment, regardless of whether they suffered further IPV or not.

Many studies indicated that, despite the intervention showing significant results, it was not possible to specifically identify what may have been associated with the improvement of the participants. In general, studies on the effectiveness of interventions show significant effects (Peuker et al., 2009). However, the literature still lacks explanations highlighting what is necessary for a treatment to be effective (Kazdin, 2007). This is mainly due to the complexity and specificities of the target population. Three studies analyzed in this review (Johnson et al., 2016; Orang et al., 2017; Trabold et al., 2017) mentioned the importance of future intervention studies with women victims of IPV being developed in different contexts and being more specific. They recommend considering limitations, clinical symptoms, and presence or absence of risk and protective factors, in order to assess the true effectiveness and verify whether the reduction of symptoms can be attributed exclusively to the proposed treatment. 


\section{Discussion}

The aim of this study was to investigate methodological aspects of the follow-up evaluations of psychotherapy protocols for women with a history of IPV, in addition to analyzing factors associated with the difficulties and challenges of carrying out these assessments. The countries in which the interventions took place were the USA $(n=4)$, Spain $(n=2)$, Portugal $(n=1)$, China $(n=1)$, Colombia $(n=1)$, India $(n=1)$, and Iran $(n=1)$. The presence of only one study in Latin America during the period and the absence of studies published in Brazil with the desired criteria should be highlighted. A systematic review that evaluated empirical scientific productions regarding IPV in Brazil, published in national journals, between 2013 and 2018, found a homogeneous distribution of articles over the years (Curia et al., 2020). However, the review found no study on psychological interventions for women with a history of IPV and highlighted a lack of research focusing on the assessment of psychological interventions, which indicates an important gap in relation to the phenomenon in Brazil. The time period defined for the databases searches in the present study was the previous 5 years, including productions from 2015 to 2020 . This interval was chosen in order to review the recent literature and provide an update regarding publications of studies on interventions for women who had experienced IPV that included a follow-up.

The majority of the studies used similar methodologies to carry out the interventions, as well as the follow-up assessments. The results showed that few studies had a control group and all used self-report scales to assess the clinical outcomes. A systematic review sought to evaluate intervention models for women with a history of IPV. The review analyzed 57 articles with this theme and found that, in most studies, samples were small and that some studies were pilots that were not replicated later (Feder et al., 2011). Randomized clinical trials (RCT) are fundamental for evaluating the effectiveness of treatments, but it is also important that they are guided by ethical principles to protect the participants. Some specific groups may require greater monitoring during the trial due to the presence of risk factors (Alexander et al., 2018). Risk factors related to the safety and integrity of the women and the need for access to interventions, due to psychological suffering, often prevent designs with control groups in these studies (Crespo \& Arinero, 2010). Deaton and Cartwright (2018) argue that the method most likely to produce good causal inference depends on what one intends to discover, and on how much of the phenomenon is already known. Depending on the focus of the study, other methods of investigation may be superior - empirical, theoretical or conceptual studies, for example.

Considering the format of the follow-up assessment, it was found that in most of the studies, the focus was on the reapplication of the base instruments of the intervention. In the studies, a prevalence of self-report scales was verified. These scales are often used due to their low cost, ease and flexibility during the application, as well as allowing unobservable behaviors to be investigated in studies (Coman \& Richardson, 2006; Kormos \& Gifford, 2014). However, self-report measures are based on people's perceptions of their functioning. Accordingly, the 
answers have subjective estimates that can influence the reliability of the data, such as personal limitations (e.g. if the person is more introspective or shy, comprehension and memory capacity) and characteristics associated with an experimental environment (Rosenman et al, 2011; Vinksi \& Watter, 2012).

Inaccuracies in self-report scales can also be associated with factors such as the "over reporting" of behavior and social desirability bias, that is, exaggerating and the tendency to respond with positive affirmations (Kormos \& Gifford, 2014). A study by Rosenman et al., (2011) indicates that, in some interventions such as psychotherapy, the participant is expected to change their conceptions over time (pretest and post-test), as they get a better understanding on the therapeutic goals, or start trusting more in the researcher, resulting in a "shift answer bias". In this sense, it is essential to verify the type and intensity of response bias at the different evaluation times to detect any shift answer bias. Furthermore, there is a question about the ability of self-report measures to detect effective changes in the functioning of the individual over time (Cress et al., 1995). The same challenge is faced by qualitative studies when categorizing their results, especially due to the dichotomous separation of "good" or "bad". A meta-synthesis of qualitative studies identified that, after psychotherapy, the participants experienced perceptual changes in their level of awareness, self-understanding, behavioral change, problem solving skills, and emotional experience (Timulak, 2007, 2010). Currently, there is a need to develop mixed method research to understand the relationship between instrument scoring and the participants' underlying experiences during the study, which may be associated with different mechanisms (De Smet et al., 2019).

Regarding the sample selection, it is important to critically analyze the inclusion and exclusion criteria of the study participants. As described in the results, most of the studies did not request that the participant was already separated from the abusive partner, maintaining active stressful situations that directly impact the conduct of the treatment and mental health outcomes (Bogat et al., 2013; Trabold et al., 2020). Experiencing IPV can trigger important long-term consequences for mental health. The majority of women manifest clinical symptoms of PTSD months after seeking help, due to the violence suffered (McFarlane et al., 2020). Consecutive situations of abuse significantly reduce the effect of treatment, culminating in difficulties in remitting symptoms of PTSD and depression, leading to an increased risk of re-victimization (Cattaneo \& Goodman, 2005; Trabold et al., 2020). Most studies available in the literature report improvements between the pre- and post-test; however, few identify significant changes at least 1 year after the intervention. One study indicated that, after an intervention with 118 women, some $(n=26)$ showed little response to the treatment and reported new experiences of IPV during the 6-month follow-up period. These participants manifested higher symptoms of depression and PTSD. Considering the results, the authors indicated the need for longer treatment with more weekly evaluations for these women. The study also highlighted the importance of including a longer follow-up period, in order to assess the impact of the treatment and the long-term risk for situations of violence in intimate relationships (Iverson et al., 2011).

These notes reveal that there is still a gap in the literature regarding adequate psychological treatment for women with a history of IPV and diagnosis of PTSD. A 
systematic review indicated the importance of the professionals that care for these women being trained in specific trauma interventions, such as Cognitive Processing Therapy and Trauma-Focused Cognitive-Behavioral Therapy (TF-CBT) (Trabold et al., 2020). Another recent study suggests culturally sensitive interventions that take into account the emotional expressions and nuances of the local culture, as they tend to be more effective in reducing the symptoms of PTSD in women with a history of IPV (Shaked et al., 2020). The study by McFarlane et al. (2020) performed a 7-year follow-up assessment with 271 women with a history of IPV. The period was chosen by the funding agency itself specifically to check for long-term PTSD symptoms and develop an optimal treatment program, as well as assist in policy decisions and resource allocations. The results indicated that more than $25 \%$ of the women reported significant clinical symptoms of PTSD 7 years after seeking help. Furthermore, they point out that not only PTSD, but also other clinical outcomes in mental health may appear later as well (McFarlane et al., 2020). Post-traumatic stress disorder is a frequent consequence of IPV and a serious disorder that can compromise the overall functioning of women (WHO, 2019). In light of this, the importance of investing in studies that develop and longitudinally evaluate interventions for PTSD specifically in the context of IPV is emphasized.

Another point to be discussed is related to access to the women for the followup assessment. Most women that participate in psychotherapeutic interventions present serious limitations, especially at work and financially, which prevent them from going to the place to carry out the assessment. Few studies discuss the obligation to conduct the assessment face-to-face or to be flexible in the access to the participants for the follow-up. When leaving an abusive relationship, women face important changes in terms of priorities and living conditions (Anderson \& Saunders, 2003). Many factors can facilitate or hinder their participation in assessments, which highlights the need for the adaptation of these assessments, especially considering the complexity and unique circumstances experienced by the women in the long term (Ford-Gilboe et al., 2020).

Some of these factors can be identified in the study by Choi et al. (2018), whose sample consisted of 100 women with a history of IPV who were sheltered in refugee centers in Hong Kong. These centers provide temporary shelter for women who urgently need to flee their homes to protect themselves from the violence of their partners. While in the shelter, they receive accommodation, food, counseling services and psychological treatment, such as the intervention carried out by Choi and colleagues (2018). These women can use some of the center's services for up to 3 months after leaving the shelter. However, the bond between the women and the professional team is often not sustained after she leaves. This can happen due to the women resuming the relationship and returning to live with the aggressor, which often culminates in no longer being able to attend the shelter. In the case that the woman ceases contact with the aggressor, changes to contact information become common, which hampers contact with the refuge center. Therefore, interruptions in the psychotherapy process may occur, as well as non-attendance in the follow-up evaluation.

The study by Johnson and colleagues (2016), performed follow-up assessments at 1 week, 3 months, and 6 months after intervention with women, residing in regional shelters $(n=60)$. Continuous treatment after shelter leave was identified as a key 
fator for therapeutics effectiveness, especially regarding PTSD symptoms worsening or remission. However, the results show that most women are unable to remain in support groups or under psychological treatment after leaving the shelter, mainly for logistical reasons. Commuting emerges as a hindrance, both due to the value of transport and the complexity of reconciling work schedules with support group attendance (Johnson et al., 2016).

Beck et al. (2016), identified no losses in the follow-up stage, carried out 1 month after the end of the intervention. However, the authors emphasize the importance of having a long-term evaluation to ensure the maintenance of the results. The authors discuss the challenges faced by women with a history of violence alongside aggression: unemployment or precarious working conditions, unstable or unhealthy housing, legal disputes over children custody (when the aggressor is the father), among others. Additionally, they highlight the impact caused by these factors on the access to mental health treatments: two participants dropped out of treatment after finding better-paying jobs whose schedule conflicted with the intervention sessions. When faced with this decision, most women end up choosing financial stability over pychotherapy (Beck et al., 2016).

The study by Muñoz et al. (2015) highlights another extremely relevant factor by focusing on women with substance abuse and who experience intimate partner violence. Substance abuse tends to increase social vulnerability, which may culminate in incarceration and psychiatric hospitalizations. These factors result in a complete halt of the ongoing psychological treatment, as well as attendance for the follow-up evaluations (Muñoz et al., 2015).

Regarding the follow-up interval, it was observed that 3 months was the period most used. Conducting follow-up assessments presents numerous obstacles, especially with long intervals (e.g. 12 months). Considering the complexity of the phenomenon of violence against women, it is important to understand external variables that can influence the participation of women in longer studies. The lack of necessary financial resources and other limitations already described can cause a total loss of communication prior to the follow-up evaluations (Simmons et al., 2015). Financial incentives in studies can be a great ally to strengthen engagement in the intervention process, being more effective than other methods (Giles et al., 2014; Giuffrida \& Torgerson, 1997). In the results, it was possible to identify four studies that described the use of financial incentives during the research. For example, the study conducted by Allard and colleagues (2018) offered US\$400 to each participant after completing all stages of the intervention, including the follow-up. The results showed that only one participant was not accessed in the follow-up. In the study by Johnson and colleagues (2016), participants were offered US\$50 for each stage completed and no losses were observed at the follow-up, in either group. It should be noted that the women in this study were residents of shelter institutions in situations of social vulnerability; therefore, small incentives could assist in the recovery process and result in greater commitment to the intervention.

Allowing follow-up sessions on less common shifts (e.g. at night or weekends) could also be an alternative to reach a larger number of participants for the assessment. However, commuting to the research facility can be another obstacle. With the pandemic situation of COVID-19, many researchers needed to readjust their studies and collection 
procedures, in order to adapt to the current moment. As an adaptation, online platforms for applying instruments, interviews or even interventions have been used, and are a promising strategy to engage a greater number of participants in long-term evaluations.

The results obtained through the studies are fundamental and offer evidence-based recommendations for the exercise of the clinical practice (Peuker et al., 2009). Studies in which psychotherapeutic interventions are performed can provide adequate and reliable information about techniques and instruments that can assist the treatment, especially with vulnerable populations (Peuker et al., 2009; Simmons et al., 2015). In the case of interventions with women with a history of IPV, there are a number of factors that can influence the treatment and that require the attention of the researcher/clinical professional. Regarding the implications for research, there is a need to develop intervention protocols capable of engaging and increasing the adherence of the participants in the study, especially in the follow-up assessments. As described in the results, there was a prevalence of studies requesting a diagnosis of current PTSD of the participant. The importance of using treatment models focused on trauma is identified, mainly in relation to safety and the development of capacities to deal with the violence suffered (Johnson et al., 2011). In this sense, follow-up assessments can also be adapted to verify risk factors after the end of the intervention, such as identifying possibilities for re-victimization and responding to the long-term impact of the trauma. Likewise, assessments can enhance protective factors that promote the reconstruction of the sense of control over one's own life and autonomy (Trabold et al., 2020). The importance of establishing and strengthening the therapeutic bond with the participant is emphasized, aiming to prevent disruptions and possible dropouts throughout the process (Peuker et al., 2009).

In the clinical practice, the professional must also investigate, in an appropriate manner, the life history, including the past and current history of IPV (e.g. length of relationship, types of violence), as well as develop an effective treatment plan. For this, it is necessary to assess the main symptoms resulting from the abusive experience (e.g. depressive, anxious or post-traumatic symptoms), to verify the presence or absence of a support network, and to use evidence-based techniques that assist the patient in the elaboration of the trauma and in the development of emotional and behavioral strategies. During the treatment, it is essential for the professional to promote engagement through the therapeutic bond, be aware of the cycle of violence against women, and develop a safety plan together with the patient (Bogat et al., 2013). It should be noted that, in the clinical context, there are specific challenges and impasses for psychotherapy that are often not translated in research (Peuker et al., 2009). Therefore, the need can be identified for studies to clearly and objectively provide data about the effectiveness of treatments for women with a history of IPV that can be used in the clinical practice.

\section{Conclusions}

The results of this study allowed the most used formats and intervals of the follow-up of psychological treatment for women with a history of IPV to be identified and critically analyzed, as well as the difficulties and challenges associated with the practice to be mapped. The most used format was the reapplication of 
the instruments used in the initial assessment, and the intervals varied, with the period of 3 months being the most reported.

From the analyses performed, some gaps were evident, such as the absence of a standardized protocol, especially for women with a history of IPV and PTSD, a symptom that often does not present satisfactory reductions in follow-up assessments. It was also identified that women with a history of IPV have complex living conditions, often marked by changes of telephone number, home address and job, which may be associated with greater dropout or the inability to locate the woman to perform the follow-up assessment. In addition, financial difficulties and the absence of trustworthy people to leave children with were also factors that affected the performance of the follow-up interview, requiring new evaluation alternatives to be developed, such as the telephone interview. There is also space to rethink possibilities of providing financial incentives for the participation in Brazilian research, since the reimbursement of the cost of the attendance could allow these women to perform the interview.

Conducting scientific studies with women with a history of IPV is delicate, as the research is traversed by ethical issues that also concern the physical and emotional security of the participants. The same phenomena encompass followup interviews, making it necessary for researchers to be aware of the woman's health status and be prepared to offer the necessary guidance and support for each patient, even after the end of the psychotherapy. With this, the importance and the need to adapt the follow-up assessments are emphasized, so that they can also evaluate risk factors as a way to reduce the negative consequences of the violence suffered, enhancing protection factors that act directly in the guarantee of the women's rights.

Author contribution Literature review, data collection, results and discussion was done by LN Foschiera and MF Dupont. Study design and final review was done by LN Foschiera and LF Habigzang.

Funding The research leading to these results received funding from the Conselho Nacional de Desenvolvimento Científico e Tecnológico (CNPq) granted to Luísa Fernanda Habigzang (research productivity level 1D).

Data availability Data sharing not applicable to this article as no datasets were generated or analyzed during the current study.

\section{Declarations}

Ethics approval and consent to participate Informed consent statement is not applicable to this article.

Competing interests The authors declare no competing interests.

\section{References}

Alexander, S., Pillay, R., \& Smith, B. (2018). A systematic review of the experiences of vulnerable people participating in research on sensitive topics. International Journal of Nursing Studies, 88, 85-96. https://doi.org/10.1016/j.ijnurstu.2018.08.013 
Allard, C. B., Norman, S. B., Thorp, S. R., Browne, K. C., \& Stein, M. B. (2018). Mid-treatment reduction in trauma-related guilt predicts PTSD and functioning following cognitive trauma therapy for survivors of intimate partner violence. Journal of Interpersonal Violence, 33(23), 3610-3629. https://doi.org/10.1177/0886260516636068

American Psychological Association. (2017, July 31). What is psychotherapy? https://www.apa.org/ ptsd-guideline/patients-and-families/psychotherapy

American Psychological Association (n.d.). Intervention. In APA dictionary of psychology. Retrieved September 10, 2021, from https://dictionary.apa.org/intervention

Anderson, D. K., \& Saunders, D. G. (2003). Leaving an abusive partner: An empirical review of predictors, the process of leaving, and psychological well-being. Trauma, Violence, \& Abuse, 4(2), 163-191. https://doi.org/10.1177/1524838002250769

Arroyo, K., Lundahl, B., Butters, R., Vanderloo, M., \& Wood, D. S. (2017). Short-term interventions for survivors of intimate partner violence: A systematic review and meta-analysis. Trauma, Violence, \& Abuse, 18(2), 155-171. https://doi.org/10.1177/1524838015602736

Bacchus, L. J., Ranganathan M., Watts C., \& Devries, K. (2018). Recent intimate partner violence against women and health: A systematic review and meta-analysis of cohort studies. BMJ Open, 8(7), Article e019995. https://doi.org/10.1136/bmjopen-2017-019995

Bardin, L. (2011). Análise de Conteúdo. [Content Analysis]. Edições 70

Beck, J. G., Tran, H. N., Dodson, T. S., Henschel, A. V., Woodward, M. J., \& Eddinger, J. (2016). Cognitive trauma therapy for battered women: replication and extension. Psychology of Violence, 6(3), 368-377. https://doi.org/10.1037/vio0000024

Berg, H. (2019). How does evidence-based practice in psychology work?-As an ethical demarcation. Philosophical Psychology, 32(6), 853-873. https://doi.org/10.1080/09515089.2019.1632424

Bogat, G. A., Garcia, A. M., \& Levendosky, A. A. (2013). Assessment and psychotherapy with women experiencing intimate partner violence: Integrating research and practice. Psychodynamic Psychiatry, 41(2), 189-217. https://doi.org/10.1521/pdps.2013.41.2.189

Cattaneo, L. B., \& Goodman, L. A. (2005). Risk factors for reabuse in intimate partner violence: A cross-disciplinary critical review. Trauma, Violence, \& Abuse, 6(2), 141-175. https://doi.org/10. $1177 / 1524838005275088$

Choi, A. W. M., Chan, P. Y., Lo, R. T. F., Wong, L. C. L., Wong, J. Y. H., \& Tang, D. H. M. (2018). Freeing Chinese abused women from stereotype: A pretest-posttest comparison study on group intervention in refuge centers. Journal of Evidence-Informed Social Work, 15(6), 599-616. https://doi.org/10.1080/23761407.2018.1509409

Cort, N. A., Cerulli, C., Poleshuck, E. L., Bellenger, K. M., Xia, Y., Tu, X., Mazzotta, C. M., \& Talbot, N. L. (2014). Interpersonal psychotherapy for depressed women with histories of intimate partner violence. Psychological Trauma: Theory, Research, Practice, and Policy, 6(6), 700-707.https://doi. org/10.1037/a0037361

Crespo, M., \& Arinero, M. (2010). Assessment of the efficacy of a psychological treatment for women victims of violence by their intimate male partner. The Spanish Journal of Psychology, 13(2), 849863. https://doi.org/10.1017/S113874160000250X

Cress, M. E., et al. (1995). Relationship between physical performance and self-perceived physical function. Journal of the American Geriatrics Society, 43(2), 93-101. https://doi.org/10.1111/j.15325415.1995.tb06372.x

Curia, B. G., Gonçalves, V. D., Zamora, J. C., Ruoso, A., Ligório, I. S., \& Habigzang, L. F. (2020). Produções Científicas Brasileiras em Psicologia sobre Violência contra Mulher por Parceiro Íntimo [Brazilian Scientific Productions in Psychology on Violence against Women by Intimate Partner]. Psicologia: Ciência e Profissão, 40, Article e189184. https://doi.org/10.1590/1982-3703003189184

Coman, L., \& Richardson, J. (2006). Relationship between self-report and performance measures of function: A systematic review. Canadian Journal on Aging, 25(3), 253-270. https://doi.org/10.1353/cja.2007.0001

Deaton, A., \& Cartwright, N. (2018). Understanding and misunderstanding randomized controlled trials (2018). Social Sciences \& Medicine, 210, 2-21. https://doi.org/10.1016/j.socscimed.2017.12.005

De Smet, M. M., Meganck, R., De Geest, R., Norman, U. A., Truijens, F., \& Desmet, M. (2019). What "good outcome" means to patients: Understanding recovery and improvement in psychotherapy for major depression from a mixed-methods perspective. Journal of Counseling Psychology. Advance online publication. https://doi.org/10.1037/cou0000362

Eckhardt, C. I., Murphy, C. M., Whitaker, D. J., Sprunger, J., Dykstra, R., \& Woodard, K. (2013). The effectiveness of intervention programs for perpetrators and victims of intimate partner violence. Partner Abuse, 4(2), 196-231. https://doi.org/10.1891/1946-6560.4.2.196 
Feder, L., Niolon, P. H., Campbell, J., Wallinder, J., Nelson, R., \& Larrouy, H. (2011). The need for experimental methodology in intimate partner violence: Finding programs that effectively prevent IPV. Violence against Women, 17(3), 340-358. https://doi.org/10.1177/1077801211398620

Ford-Gilboe, M., Varcoe, C., Scott-Storey, K., Perrin, N., Wuest, J., Wathen, C. N., Case, J., \& Glass, N. (2020). Longitudinal impacts of an online safety and health intervention for women experiencing intimate partner violence: Randomized controlled trial. BMC public health, 20(1). https://doi.org/ 10.1186/s12889-020-8152-8

Giles, E. L., Robalino, S., McColl, E., Sniehotta, F. F., \& Adams, J. (2014). The effectiveness of financial incentives for health behaviour change: Systematic review and meta-analysis. PLOS ONE, 9(3), Article e90347. https://doi.org/10.1371/journal.pone.0090347

Giuffrida, A., \& Torgerson, D. J. (1997). Should we pay the patient? Review of financial incentives to enhance patient compliance. BMJ, 315(7110), 703-707. https://doi.org/10.1136/bmj.315.7110.703

Kormos, C., \& Gifford, R. (2014). The validity of self-report measures of proenvironmental behavior: A meta-analytic review. Journal of Environmental Psychology, 40, 359-371. https://doi.org/10.1016/j. jenvp.2014.09.003

Habigzang, L. F., Schneider, J. A., Frizzo, R. P., \& Freitas, C. P. P. (2018). Evaluation of the impact of a cognitive-behavioral intervention for women in domestic violence situations in Brazil. Universitas Psychologica, 17(3), 1-11. https://doi.org/10.11144/Javeriana.upsy17-3.eicb

Hameed, M., O’Doherty, L., Gilchrist, G., Tirado-Muñoz, J., Taft, A., Chondros, P., Feder, G., Tan, M., \& Hegarty, K. (2020). Psychological therapies for women who experience intimate partner violence. Cochrane Database of Systematic Reviews. https://doi.org/10.1002/14651858.CD013017.pub2

Hardesty, J. L., \& Ogolsky, B. G. (2020). A socioecological perspective on intimate partner violence research: A decade in review. Journal of Marriage and Family, 82(1), 454-477. https://doi.org/10. 1111/jomf.12652

Iverson, K. M., Gradus, J. L., Resick, P. A., Suvak, M. K., Smith, K. F., \& Monson, C. M. (2011). Cognitive-behavioral therapy for PTSD and depression symptoms reduces risk for future intimate partner violence among interpersonal trauma survivors. Journal of Consulting and Clinical Psychology, 79(2), 193-202. https://doi.org/10.1037/a0022512

Johnson, D. M., Johnson, N. L., Perez, S. K., Palmieri, P. A., \& Zlotnick, C. (2016). Comparison of adding treatment of PTSD during and after shelter stay to standard care in residents of battered women's shelters: Results of a randomized clinical trial. Journal of Traumatic Stress, 29(4), 365-373.https:// doi.org/10.1002/jts. 22117

Johnson, D. M., Zlotnick, C., \& Perez, S. (2011). Cognitive-behavioral treatment of PTSD in residents of battered women shelters: Results of a randomized clinical trial. Journal of Consulting and Clinical Psychology, 79(4), 542-551. https://doi.org/10.1037/a0023822

Kazdin, A. E. (2007). Mediators and mechanisms of change in psychotherapy research. Annual Review of Clinical Psychology, 3, 1-27. https://doi.org/10.1146/annurev.clinpsy.3.022806.091432

Matud, M. P., Fortes, D., \& Medina, L. (2014). Eficacia de un programa de tratamiento psicológico individual para mujeres maltratadas por su pareja [Efficacy of an individual psychological treatment for battered women]. Psychosocial Intervention, 23(3), 199-207. https://doi.org/10.1016/j.psi.2014.06. 001

Matud, M. P., Padilla, V., Medina, L., \& Fortes, D. (2016). Eficacia de un programa de intervención para mujeres maltratadas por su pareja. Terapia Psicológica, 34(3), 199-208. https://doi.org/10.4067/ S0718-48082016000300004

McFarlane, J., Maddoux, J., Paulson, R., Symes, L., \& Jouriles, E. N. (2020). An evidence-based assessment tool for estimating future post-traumatic stress disorder: A 7-year follow-up study. Journal of Women's Health, 29(4), 520-523. https://doi.org/10.1089/jwh.2019.7699

Melnik, T., de Souza, W. F., \& de Carvalho, M. R. (2014). A importância da prática da psicologia baseada em evidências: aspectos conceituais, níveis de evidência, mitos e resistências [The importance of evidence-based practices in psychology: Conceptual aspects, evidence levels, myths and resistance]. Revista Costarricense de Psicología, 33(2), 79-92. https://www.redalyc.org/articulo.oa?id= 476747238008

Naismith, I., Ripoll, K. \& Pardo, V.M. (2020). Group compassion-based therapy for female survivors of intimate-partner violence and gender-based violence: a pilot study. Journal of Family Violence, 36, 175-182. https://doi.org/10.1007/s10896-019-00127-2

Orang, T., Ayoughi, S., Moran, J. K., Ghaffari, H., Mostafavi, S., Rasoulian, M., \& Elbert, T. (2017) The efficacy of narrative exposure therapy in a sample of Iranian women exposed to ongoing intimate 
partner violence-A randomized controlled trial. Clinical Psychology \& Psychotherapy, 25(6), 827841.https://doi.org/10.1002/cpp.2318

Patel, A., Weobong, B., Patel, V., \& Singla, D. R. (2019). Psychological treatments for depression among women experiencing intimate partner violence: findings from a randomized controlled trial for behavioral activation in Goa, India. Archives of Women's Mental Health, 22, 779-789. https://doi. org/10.1007/s00737-019-00992-2

Peuker, A. C., Habigzang, L. F., Koller, S. H., \& Araujo, L. B. (2009). Avaliação de processo e resultado em psicoterapias: Uma revisão [Assessment of psychotherapy process and outome: A review]. Psicologia Em Estudo, 14(3), 439-445. https://doi.org/10.1590/S1413-73722009000300004

Petersen, M. G. F., Zamora, J. C., Fermann, I. L., Crestani, P. L., \& Habigzang, L. F. (2019). Psicoterapia cognitivo-comportamental para mulheres em situação de violência doméstica: Revisão sistemática [Cognitive behavioral therapy for women in domestic violence situation: Systematic review]. Psicologia Clínica, 31(1), 145-165. https://doi.org/10.33208/PC1980-5438v0031n01A07

Rosenman, R., Tennekoon, V., \& Hill, L. G. (2011). Measuring bias in self-reported data. International Journal of Behavioural and Healthcare Research, 2(4), 320-332. https://doi.org/10.1504/IJBHR. 2011.043414

Rousseau, D. M., \& Gunia, B. C. (2016). Evidence-based practice: The psychology of EBP implementation. Annual Review of Psychology, 67, 667-692. https://doi.org/10.1146/annur ev-psych-122414-033336

Santos, A., Matos, M., \& Machado, A. (2017). Effectiveness of a group intervention program for female victims of intimate partner violence. Small Group Research, 48(1), 34-61. https://doi.org/10.1177/ 1046496416675226

Simmons, C. A., Lindsey, L., Delaney, M. J., Whalley, A., \& Beck, J. G. (2015). Real-world barriers to assessing and treating mental health problems with IPV survivors: A qualitative study. Journal of Interpersonal Violence, 30(12), 2067-2086. https://doi.org/10.1177/0886260514552275

Shaked, O., Dekel, R., Ben-Porat, A., \& Itzhaky, H. (2020). Predicting changes in PTSD and depression among female intimate partner violence survivors during shelter residency: A longitudinal study. Psychological Trauma: Theory, Research, Practice, and Policy, 13(3), 349-358. https://doi.org/10. $1037 /$ tra0000585

Timulak, L. (2007). Identifying core categories of client-identified impact of helpful events in psychotherapy: A qualitative meta-analysis. Psychotherapy Research, 17, 310-320. https://doi.org/10.1080/ 10503300600608116

Timulak, L. (2010). Significant events in psychotherapy: An update of research findings. Psychology and Psychotherapy: Theory, Research and Practice, 83, 421-447. https://doi.org/10.1348/147608310X 499404

Tirado-Muñoz, J., Gilchrist, G., Lligoña, E., Gilbert, L., \& Torrens, M. (2015). A group intervention to reduce intimate partner violence among female drug users. Results from a randomized controlled pilot trial in a community substance-abuse center. Adicciones, 27(3), 168-78. https://pubmed.ncbi. nlm.nih.gov/26437311/

Trabold, N., O’Malley, A., Rizzo, L., \& Russell, E. (2017). A gateway to healing: A communitybased brief intervention for victims of violence. Journal of Community Psychology, 46(2), 418428.https://doi.org/10.1002/jcop.21948

Trabold, N., McMahon, J., Alsobrooks, S., Whitney, S., \& Mittal, M. (2020). A systematic review of intimate partner violence interventions: State of the field and implications for practitioners. Trauma, Violence, \& Abuse, 21(2), 311-325. https://doi.org/10.1177/1524838018767934

Vinski, M. T., \& Watter, S. (2012). Priming honesty reduces subjective bias in self-report measures of mind wandering. Journal of Consciousness and Cognition, 21, 451-455.

World Health Organization (2019) Violence against women Intimate partner and sexual violence against women. Retrieved October 14, 2020, from https://apps.who.int/iris/bitstream/handle/10665/329889/ WHO-RHR-19.16-eng.pdf?ua=1

World Health Organization. (2020, March). COVID-19 and violence against women: What the health sector/system can do. https://www.who.int/reproductivehealth/publications/emergencies/COVID19-VAW-full-text.pdf?ua=1

Zancan, N., \& Habigzang, L. F. (2018). Regulação Emocional, Sintomas de Ansiedade e Depressão em Mulheres com Histórico de Violência Conjugal [Emotional regulation, symptoms of anxiety and depression in women with a history of intimate partner violence]. Psico-USF, 23(2), 253-265. https://doi.org/10.1590/1413-82712018230206 